\title{
Asymmetric Shaped-Pattern Synthesis for Planar Antenna Arrays
}

\author{
T. M. Bruintjes, A. B. J. Kokkeler, and G. J. M. Smit \\ Department of EEMCS, University of Twente, 7500 AE Enschede, Netherlands \\ Correspondence should be addressed to T. M. Bruintjes; t.m.bruintjes@utwente.nl
}

Received 7 October 2015; Revised 23 December 2015; Accepted 28 December 2015

Academic Editor: Lei Yu

Copyright (C) 2016 T. M. Bruintjes et al. This is an open access article distributed under the Creative Commons Attribution License, which permits unrestricted use, distribution, and reproduction in any medium, provided the original work is properly cited.

\begin{abstract}
A procedure to synthesize asymmetrically shaped beam patterns is developed for planar antenna arrays. As it is based on the quasianalytical method of collapsed distributions, the main advantage of this procedure is the ability to realize a shaped (null-free) region with very low ripple. Smooth and asymmetrically shaped regions can be used for Direction-of-Arrival estimation and subsequently for efficient tracking with a single output (fully analog) beamformer.
\end{abstract}

\section{Introduction}

As part of a next generation indoor communication infrastructure [1], adaptive antenna arrays are employed to mitigate the high losses that are experienced in the $60 \mathrm{GHz}$ [2] frequency band. Typically, adaptive arrays [3] operate by sampling the individual antennas. At high frequencies, however, this is costly and energy hungry. Fully analog beamformers could alleviate this problem by combining the signals already in the analog domain [4]. At the same time, however, the use of such a single output beamformer makes utilization of conventional adaptive array algorithms impossible or inefficient [5].

It was shown in [5] that tracking of mobile wireless devices can be accomplished efficiently by using the antenna pattern's shape. This approach requires that asymmetrically shaped beam patterns are synthesized to estimate the Direction-of-Arrival (DoA). The techniques presented in [5] are applicable to linear antenna arrays only, which have limited practical use. In this paper, an extension to planar arrays is presented. Most notably this comprises a procedure (based on the principle of collapsed distributions) to synthesize beam patterns suitable for tracking. Such beam patterns are both asymmetrical and smooth (i.e., feature a small ripple in the shaped region).

Because tracking requires that the shaped beam patterns are steered, an array geometry with high rotational symmetry is needed. The Standard Hexagonal Array (SHA) [6] suits this requirement well. However, for this geometry, existing synthesis methods were found to be inadequate given the requirements of the tracking patterns. Biologically inspired numerical algorithms, such as Genetic Algorithms [7] and Particle Swarm Optimization [8], are considered to be particularly suitable for planar array pattern synthesis. In spite of these methods being highly generic, they were found to be impractical in this case. Appendix A discusses this in more detail. The alternating projections method could also be considered. However, while the alternating projections work reasonably well (in terms of ripple performance) for linear asymmetric patterns [9], it is unknown if that is also true for planar arrays. The same applies to the least squares solutions in $[10,11]$.

This paper presents an analytical procedure based on collapsed distributions. Although collapsing a planar array distribution is a known technique $([12,13])$, only the rectangular grid array structure has been considered before, and the pattern shape had to be quadrant symmetric. The procedure is now generalized such that it does not require symmetry. Rectangular and triangular grids are supported while the shape of the array can be chosen arbitrarily (including the hexagonal shape).

Outline. The outline of this paper is as follows. First, the shaped-pattern tracking mechanism for linear antenna arrays 


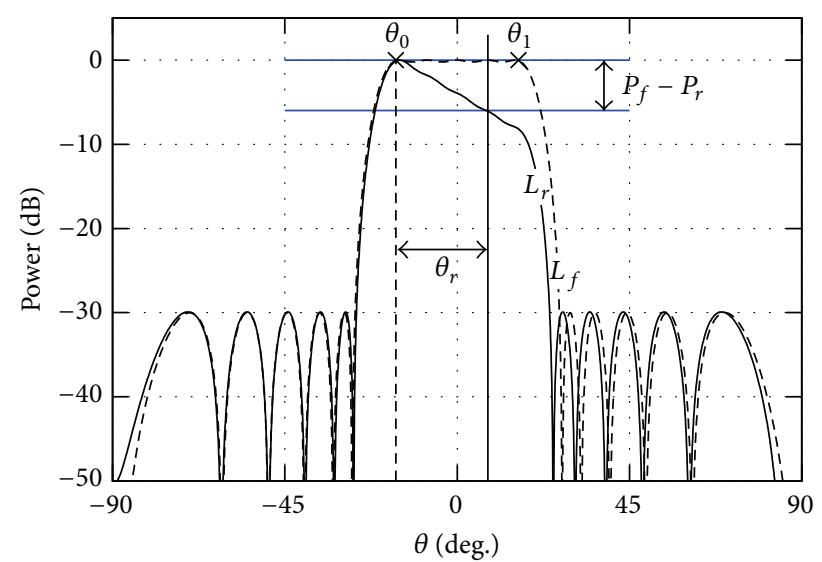

FIGURE 1: Shaped beam patterns of a 16-element equispaced linear array. The flat-top pattern $\left(L_{f}\right)$ is used to normalize the received power at a given point in time while the ramp-shaped pattern $\left(L_{r}\right)$ is used to relate the power to an angle.

is briefly recapitulated in Section 2. Section 3 explains the steps needed for extension to planar antenna arrays and why the hexagonal planar array architecture is preferred. Subsequently, the generalized collapsed distribution flow is explained in Section 4. The particularities of asymmetric pattern synthesis follow in Section 5. Steering of the asymmetric patterns, for tracking purposes, is detailed in Section 6. Future work is discussed in Section 7 and the paper is summarized in Section 8.

\section{Shaped-Pattern Tracking}

Shaped-pattern tracking concerns keeping the pattern's Main Response Axis (MRA) [6] aligned with the location of the mobile peer device. To accomplish this, DoA estimation is performed based on an asymmetrically shaped antenna pattern. If the pattern's shape is asymmetric and sufficiently predictable (i.e., smooth), such as $L_{r}$ plotted in Figure 1, the angle of incidence can be derived from the power at the output of the beamformer. This eliminates the need for crosscorrelating the individual antenna signals (as required by conventional DoA techniques [14-16]), as well as the need for exhaustive scanning [5]. However, absolute power is not a reliable measure for the DoA due to, for example, path loss or fading. A second, differently shaped, pattern is necessary to isolate power changes caused by angular displacement. In [5], a flat-top beam $L_{f}$ that overlaps with the ramp was chosen to provide reference measurements. A reliable DoA estimate can be obtained based on the power difference between $L_{r}$ and $L_{f}$ responses (i.e., $P_{f}-P_{r}$ ). To this end, let the slope of $L_{r}$ be characterized between the angles $\theta_{0}$ and $\theta_{1}$. Since $P_{\max }=L_{r}\left(\theta_{0}\right)$ and $P_{\min }=L_{r}\left(\theta_{1}\right)$ are then known values, the angular position relative to $\theta_{0}$ can be found by

$$
\theta_{r}=\frac{\left|P_{f}-P_{r}\right|}{\left|P_{\max }-P_{\min }\right| /\left(\theta_{1}-\theta_{0}\right)} .
$$

The angle $\theta_{0}$ is also known from synthesizing the patterns, so an absolute measure of the DoA is obtained by $\theta_{0}+\theta_{r}$. Finally, the MRA of $L_{f}$ and $L_{r}$ can be steered to keep the beam aligned with the location of the mobile device, giving full tracking capabilities to a single output beamformer.

\section{Extending Shaped-Pattern Tracking to Planar Antenna Arrays}

For linear antenna arrays, the response is usually evaluated only in the elevation $(\theta)$ plane directly above the axis over which the antenna elements are placed:

$$
F(\theta)=\sum_{n=1}^{N} I_{n} e^{j k x_{n} \sin (\theta)} .
$$

Herein, $x_{n}$ is the position of the $n$th element, $I_{n}$ is the (complex) excitation of that element, and $k=2 \pi$ when $x_{n}$ is expressed in units of $0.5 \lambda$ [6]. To obtain a more complete picture, the array's response should be evaluated in the full azimuth $(\phi)$ elevation coordinate system (azimuth defined as $0^{\circ}, \ldots, 360^{\circ}$ with respect to the positive $x$-axis and elevation defined as $-90^{\circ}, \ldots, 90^{\circ}$ with respect to the positive $z$-axis):

$$
F(\phi, \theta)=\sum_{n=1}^{N} I_{n} e^{j k \sin (\theta)\left(x_{n} \cos (\phi)+y_{n} \sin (\phi)\right)} .
$$

When $L_{r}$ is evaluated by (3), it will become clear that a linear antenna array will give limited tracking capabilities due to the lack of directivity. A planar array [6] provides shaping and steering capabilities in the complete $(\phi, \theta)$-space.

3.1. Planar Array Tracking Patterns. To make use of the planar array's directivity, beam shapes need to be defined which can estimate both the $\phi$ and $\theta$ components of the DoA. A set of three shapes is proposed. For two of these shapes, Figure 2 gives a 3-dimensional impression. The first beam shape, denoted as $S_{f}$, prescribes constant power over the entire $\left(\Delta \theta_{x}\right.$ by $\left.\Delta \theta_{y}\right)$ null-free region. The purpose of $S_{f}$, completely analogous to its linear counterpart, is to normalize the power level for DoA estimation. The second shape shown describes a ramp surface $S_{r y}$ directly below $S_{f}$. This ramp gives rise to the $\theta$ component with respect to the $y$-axis, and hence the $S_{r y}$ designation. A third shape is needed to estimate both the $\theta$ and the $\phi$ angle. (A smooth (synthesizable) surface below $S_{f}$, with at least one point intersecting $S_{f}$ and one point intersecting $S_{f}-\Delta P$ (Figure 2) and in which the power is unique to each $\phi$ and $\theta$ combination, does not exist.) This third shape, not shown in the figure, will be a $90^{\circ}$ rotated version of $S_{r y}$. Due to its perpendicularly oriented ramp (i.e., $\phi+90$ ), it will be referred to as $S_{r x}$.

For all synthesis purposes, the shapes are defined with respect to the zenith (steering is performed in a later stage). Whilst the center of the beams points towards $\left(0^{\circ}, 0^{\circ}\right)$, the relation between pattern shape and $\phi$ or $\theta$ might not immediately be clear. One might find it helpful to consider the silhouettes of $S_{f}$ and $S_{r y}$ at the positive $x$-axis (Figure 2). The gray area represents (an ideally shaped) $S_{r y}$ pattern steered 


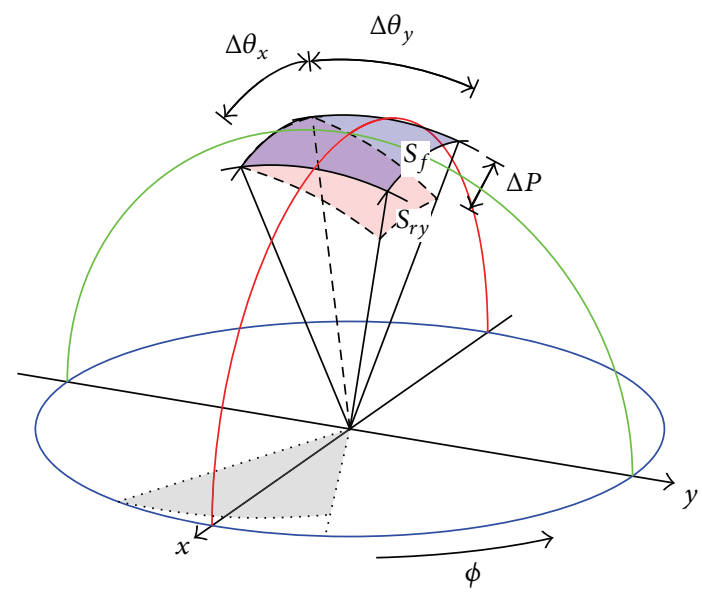

FIGURE 2: 2D tracking shapes $S_{f}$ and $S_{r y}$ visualized in $(\phi, \theta)$.

to the extreme angle $\left(0^{\circ}, 90^{\circ}\right)$. In this particular situation, $S_{r y}$ provides information about the $\phi$ angle. If the MRA is steered to $\left(90^{\circ}, 90^{\circ}\right)$, it will be $S_{r x}$ that captures $\phi$ whereas the $\theta$ angle can be found with $S_{r y}$. Thus, the role of the patterns will change as the steering angle is adjusted.

3.2. Array Configurations and Steering. Given that all three required patterns are separable (i.e., a product of two linear array patterns) [6], they could mathematically be realized fairly easily with a Standard Rectangular Array (SRA) [6]. The response of such an array can be expressed by

$$
F(\phi, \theta)=\sum_{m=1}^{M} \sum_{n=1}^{N} I_{m n} e^{j k \sin (\theta)\left(m d_{x} \cos (\phi)+n d_{y} \sin (\phi)\right)},
$$

with the excitations $I_{m n}$ of the desired 2D patterns found by multiplication

$$
I_{m n}=I_{m} I_{n}
$$

Combining, for example, the flat-top and ramp-shaped patterns from Figure 1 produces the $S_{r y}$ pattern shown in Figure 3. An asymmetrically shaped region with very low ripple can be observed, as per requirement.

To steer the pattern, let $\left(\phi_{d}, \theta_{d}\right)$ denote the desired MRA position. Steering to $\left(\phi_{d}, \theta_{d}\right)$ is then accomplished by

$$
I_{m n}=\stackrel{\circ}{I}_{m n} e^{-j k\left(x_{m} \sin \left(\theta_{d}\right) \cos \left(\phi_{d}\right)+y_{m} \sin \left(\theta_{d}\right) \sin \left(\phi_{d}\right)\right)},
$$

where $\stackrel{\circ}{I}_{m n}$ denotes the value of the $m n$th excitation before steering.

Figure 4 illustrates what happens to the shape of the $S_{f}$ pattern when it is steered to $\left(0^{\circ}, 35^{\circ}\right)$. Note in particular that the shaped region's angular coverage (indicated by the dashed lines) changes from square to rectangle. Although the effect is only shown for $S_{f}$ (as it lends itself better for illustration purposes), $S_{r x}$ and $S_{r y}$ experience the same kind of broadening in the general direction of the desired steering angle $\phi_{d}$. More severe warping of the shape occurs when the pattern is also steered away from the principal $x$ - and

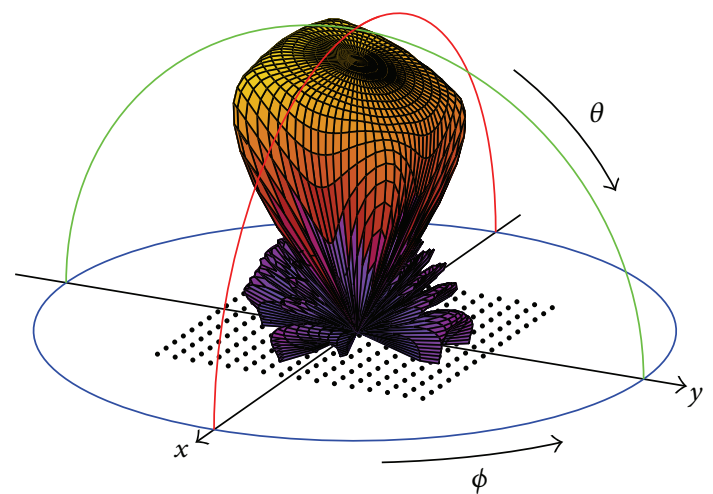

FIGURE 3: SRA-256 pattern shaped as $S_{r y}$.

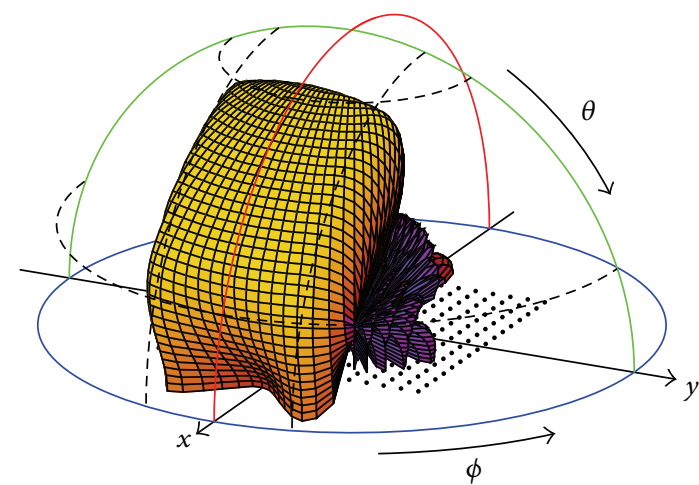

FIGURE 4: Steering the SRA-256 $S_{f}$ pattern over the principal $x$-axis $\left(0^{\circ}, 35^{\circ}\right)$ broadens its shaped region in that direction.

$y$-axes. Figure 5 depicts the $S_{f}$ pattern steered to $\left(50^{\circ}, 35^{\circ}\right)$. The angular coverage of the pattern now starts to resemble a diamond (equilateral quadrilateral) shape. This is problematic for shaped-pattern tracking. Without consistency in the shapes used for DoA estimation, the angular position of the mobile device being tracked is prone to end up in the sidelobe region due to erroneous steering. The latter is precisely what the use of shaped patterns had to prevent. To tackle this problem, the use of hexagonal arrays is proposed.

3.3. Hexagonal Planar Arrays. Figure 6 shows the 169element hexagonal array (SHA-169) that is used as an example throughout the remainder of the paper. Indexing of the elements is done in a fashion similar to the SRA, the difference being that not every row has the same number of elements. The response of this array is therefore denoted more generic

$$
F(\phi, \theta)=\sum_{m} \sum_{n} I_{m n} e^{j k \sin (\theta)\left(x_{m n} \cos (\phi)+y_{m n} \sin (\phi)\right)},
$$

with $\left(x_{m n}, y_{m n}\right)$ the position of the $m n$th element expressed with two values of $0.5 \lambda$ units. This array structure is particularly suitable for shaped-pattern tracking because of its truly equispaced antenna positions and its 6 -fold rotational symmetry (4-fold for the SRA). The equal $0.5 \lambda$ spacing ensures minimal broadening of the pattern's shaped region 


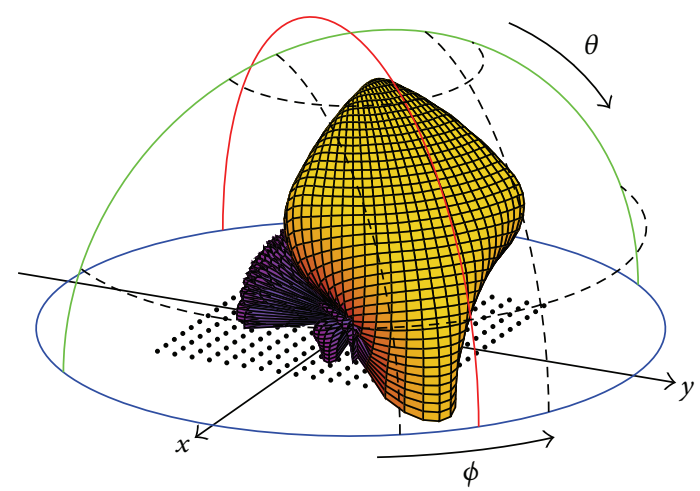

FIGURE 5: Steering $S_{f}$ off the principal axes $\left(50^{\circ}, 35^{\circ}\right)$ results in an even more severe warping of the shaped region. The boundaries of the shaped region are also wrongly oriented, leading to a different angular coverage.

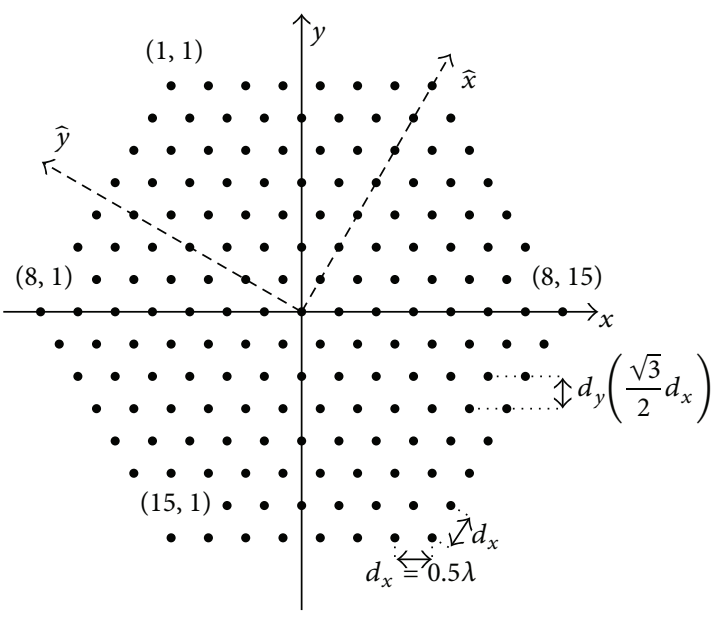

Figure 6: The Standard Hexagonal Array features equally spaced antenna elements and multiple pseudo-principal axes.

for steering angles in between the principal axis. The rotational symmetry is beneficial for the orientation (i.e., the shape of the null-free region) of the patterns. For the latter, assume that a pattern steered to the zenith has a particular orientation with respect to the $x$ and $y$ reference axes. By rearranging the array excitations, this orientation can be changed to any of the rotational symmetric axes (e.g., $\hat{x}$ and $\hat{y}$ in Figure 6), without affecting the pattern's shape. Such axes will be referred to as pseudo axes. The hexagonal array is rotationally symmetric every $60^{\circ}$ whereas this is only the case every $90^{\circ}$ with the SRA. Moreover, when it is considered that for all practical purposes $S_{r x}$ and $S_{r y}$ are equivalent when rotated $90^{\circ}$, the beam pattern can be reproduced exactly every $30^{\circ}$. This greatly reduces the need for off-axis $\phi$ steering.

The downside of the SHA geometry is that the array excitations are no longer separable. Synthesizing the shaped patterns is therefore much more difficult.

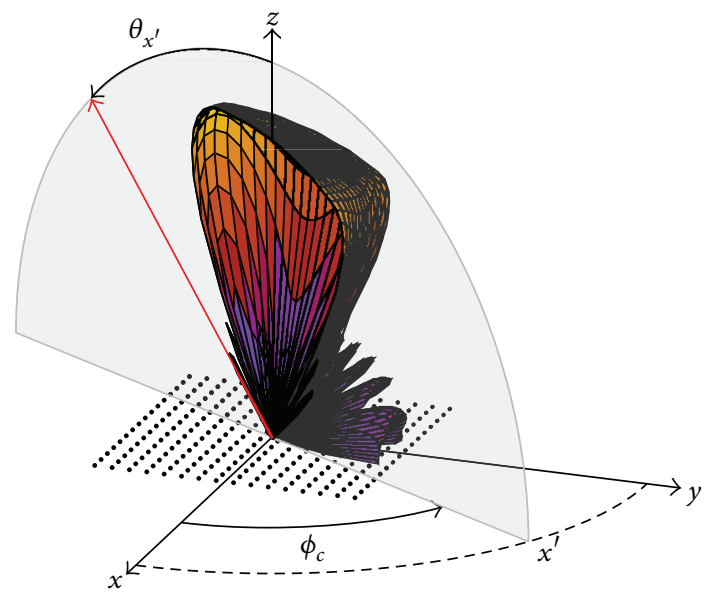

FIGURE 7: The response of a collapsed equivalent linear array is identical to the planar array's response in the $x^{\prime} z$-plane (i.e., a $\phi$ cut), shown here for the $16 \times 16$ SRA $P_{r y}$ pattern, evaluated in $\phi_{c}=63.4349^{\circ}$.

\section{Collapsed Distributions}

In this paper, the quasi-analytical method of collapsed distributions is utilized. With the right adjustments, this technique excels at the synthesis of low ripple asymmetrically shaped beams. It also matches well with our work for linear arrays, presented earlier in $[5,17]$. The general idea of collapsed distributions is to reduce the planar array geometry to an equivalent linear array, using a coordinate transformation. It is then possible to synthesize, using linear array techniques, a shape for the $\phi_{c}$-cut that corresponds to the $\phi$ angle over which the array was collapsed. Figure 7 illustrates this procedure. In order to synthesize a planar array pattern, the procedure must be repeated for several $\phi_{c}$-cuts. The next step is then to reverse the collapsing and spread the excitations over the original planar array. Synthesis based on collapsed distributions therefore involves the following actions:

(1) collapsing (projecting) the SHA-169 array to a particular azimuth angle $\phi_{c}$, to obtain its equivalent collapsed linear array;

(2) invoking a linear array synthesis algorithm to synthesize the $1 \mathrm{D}$ shape dictated by the $\phi_{c}$ crosscut of a desired 2D pattern shape (Figure 7);

(3) repeating step (2) for multiple $\phi_{c}$ angles;

(4) carrying out adjustments on the excitations from step (2) as needed (Section 5);

(5) reversing the collapsing process by spreading the excitations to their original planar distribution.

This procedure only synthesizes patterns pointing to the zenith. Steering the patterns will be dealt with separately, after step (5). 


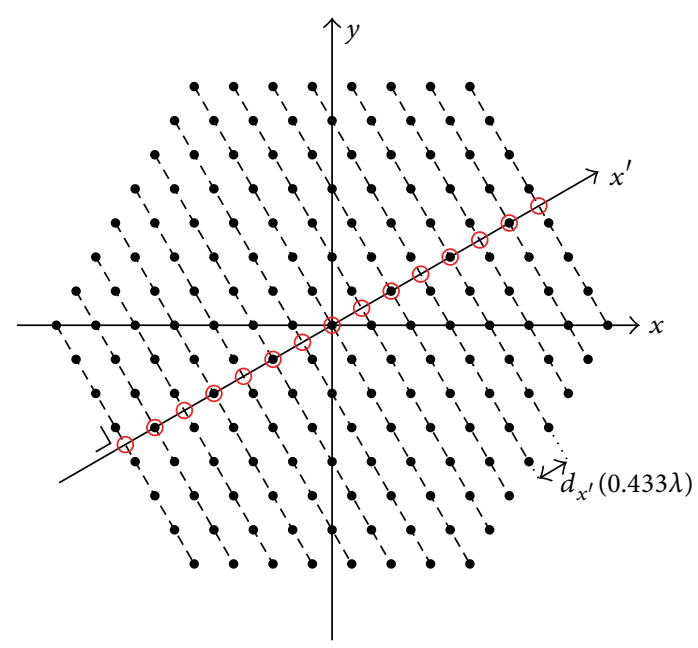

FIgURE 8: Collapsing the Standard Hexagonal Array-169 (with $0.5 \lambda$ spacing) onto $\phi_{c}=30^{\circ}$ yields a $0.433 \lambda$ equispaced linear array of 15 elements. The element positions are indicated by the positions marked on the $x^{\prime}$-axis.

4.1. Collapsing the Planar Array. To collapse an arbitrary planar array, a common rotation in the $x y$-plane must first be applied to all the positions of the array elements. Let the position of the $m n$th element from (4) be denoted in vector notation by $\left[\begin{array}{ll}x_{m n} & y_{m n}\end{array}\right]^{T}$. The rotation angle $\alpha\left(0^{\circ} \leq \alpha \leq 360^{\circ}\right)$ is defined with respect to the positive $x$-axis and the rotation itself by

$$
\left[\begin{array}{c}
x_{m n}^{\prime} \\
y_{m n}^{\prime}
\end{array}\right]=\left[\begin{array}{cc}
\cos (\alpha) & -\sin (\alpha) \\
\sin (\alpha) & \cos (\alpha)
\end{array}\right]\left[\begin{array}{l}
x_{m n} \\
y_{m n}
\end{array}\right]
$$

where $\left(x_{m n}^{\prime}, y_{m n}^{\prime}\right)$ is the element position after rotation. Based on this transformation, (7) can be rewritten into

$$
F(\phi, \theta)=\sum_{m} \sum_{n} I_{m n} e^{j k \sin (\theta)\left(x_{m n}^{\prime} \cos (\phi-\alpha)+y_{m n}^{\prime} \sin (\phi-\alpha)\right)},
$$

which becomes interesting when the special condition $\alpha=\phi$ is met. Under this condition, (9) reduces to

$$
F\left(\theta_{x^{\prime}}\right)=\sum_{m} \sum_{n} I_{m n} e^{j k x_{m n}^{\prime} \sin \left(\theta_{x^{\prime}}\right)}
$$

where $\theta_{x^{\prime}}$ is the elevation angle $\theta$ measured in the $x^{\prime} z$-plane (see Figure 7). This means that, for $\alpha=\phi$, the $y^{\prime}$-position of the antenna elements becomes irrelevant. Each element is thus projected (i.e., collapsed) onto the $x^{\prime}$-axis, over a line perpendicular to the $x^{\prime}$-axis. Figure 8 depicts this projection graphically.

Note that (10) is equivalent to (2), that is, the array factor of a linear antenna array. This holds for conventional patterns, but also for more complicated cases such as shaped patterns [18]. The equivalence makes collapsing a planar array interesting, because synthesizing a particular shape for (10) is a well understood problem.
TABLE 1: Collapse angles for triangular grid arrays. $L$ is the number of elements for the proposed 169-element SHA.

\begin{tabular}{lcc}
\hline$\phi_{c}$ & $d_{x^{\prime}}$ & $L$ \\
\hline $0^{\circ}$ & $0.25 \lambda$ & 29 \\
$10.89^{\circ}$ & $0.1636 \lambda$ & 43 \\
$16.10^{\circ}$ & $0.121 \lambda$ & 57 \\
$19.11^{\circ}$ & $0.09446 \lambda$ & 71 \\
$30^{\circ}$ & $0.433 \lambda$ & 15 \\
$40.89^{\circ}$ & $0.09446 \lambda$ & 71 \\
$43.90^{\circ}$ & $0.121 \lambda$ & 57 \\
$49.11^{\circ}$ & $0.1636 \lambda$ & 43 \\
$60^{\circ}$ & $0.25 \lambda$ & 29 \\
$70.89^{\circ}$ & $0.1636 \lambda$ & 43 \\
$76.10^{\circ}$ & $0.121 \lambda$ & 57 \\
$81.05^{\circ}$ & $0.07779 \lambda$ & 85 \\
$90^{\circ}$ & $0.433 \lambda$ & 15 \\
\hline
\end{tabular}

4.2. Distribution Angles. Linear array synthesis techniques are numerous and diverse. Due to this versatility, one could theoretically collapse the planar array for any given $\phi$. In practice, however, there are various restrictions that need to be considered in order to be able to, later on, reverse the process properly by spreading out the collapsed distributions. In essence, two factors need to be taken into consideration before choosing a $\phi_{c}$ angle. Firstly, the resulting collapsed distribution and the linear array synthesis method to be used must be compatible. Secondly, the $\phi_{c}$ angles should preferably be distributed evenly over the azimuth, to control the shape of the pattern as good as possible.

The Orchard-Elliott [19] procedure is a proven method to synthesize patterns with a low ripple shaped region [5] and is therefore used to find the excitations for the collapsed distributions. This method requires an equispaced linear array. For any planar array with its element positions arranged in a triangular or rectangular grid [18], this requirement is met by collapsing the array over selected angles. The relevant angles for rectangular grid arrays have been discussed in [13]. The selected angles that produce an equispaced linear array for triangular grids (regardless of its shape) have been listed in Table 1. For each of these $\phi_{c}$ angles, a similar collapsed distribution can also be found at $\phi_{c}+90^{\circ}$. However, in this paper these will not be needed because the desired shapes are still symmetric over one axis.

4.3. Linear Array Synthesis. Once the collapsed distributions are formed, one can start synthesizing the desired linear shape for the corresponding $\phi$ angle. For the example from Figure 2, linear shape definitions will be formalized to this end. Let the slope of the ramp $\Delta P / \Delta \theta$ be set to $-10 \mathrm{~dB} / 40^{\circ}$ such that the power differences used for DoA estimation will be similar to those used in [5]. Likewise, the height of the sidelobes is set to $-30 \mathrm{~dB}$ outside the shaped region. As mentioned before, $\Delta \theta$ will be kept $\phi$ invariant. Combining these constraints, a 
shaping contour $S\left(\phi_{c}, \theta\right)$ can be derived for an arbitrary $\phi_{c}$ angle

$$
\begin{aligned}
& S_{f}\left(\phi_{c}, \theta\right)= \begin{cases}0, & \text { if }|\theta| \leq 20^{\circ}, \\
-30, & \text { if }|\theta|>20^{\circ},\end{cases} \\
& S_{r x}\left(\phi_{c}, \theta\right) \\
& \quad= \begin{cases}-\frac{1}{4} \theta\left(1-\frac{\phi_{c}}{90^{\circ}}\right), & \text { if }|\theta| \leq 20^{\circ}, 0^{\circ} \leq \phi_{c} \leq 90^{\circ}, \\
-30, & \text { if }|\theta|>20^{\circ},\end{cases} \\
& S_{r y}\left(\phi_{c}, \theta\right)= \begin{cases}-\frac{1}{4} \theta\left(\frac{\phi_{c}}{90^{\circ}}\right), & \text { if }|\theta|, 0^{\circ} \leq \phi_{c} \leq 90^{\circ}, \\
-30, & \text { if }|\theta|>20^{\circ} .\end{cases}
\end{aligned}
$$

Note that, in these definitions, $S$ is always zero at $\theta=0^{\circ}$. This accommodates a common normalization of the collapsed distribution responses, to be discussed later.

Since the $\phi$-cuts are shaped by Orchard-Elliott synthesis, the array factor will be expressed in the Schelkunoff unit circle representation [20]:

$$
F(w)=I_{N} \prod_{n=1}^{N-1}\left(w-w_{n}\right) .
$$

The beamwidth is largely determined by the number of roots $\left(w_{n}\right)$ in (12) that are positioned off the unit circle. Table 1 shows that the visible region is frequently smaller than $180^{\circ}$ (i.e., $d_{x^{\prime}}<0.5 \lambda$ ). One might expect that this needs to be reflected in the number of roots placed off the unit circled. However, the visible region (and thus region I) is proportional to the number of elements in the collapsed distribution. Due to this almost fixed ratio, always four roots need to be placed off the unit circle to create a null-free region of roughly $40^{\circ}$. The shape of the antenna array then dictates the resulting shaped region boundaries. Forcing the null-free region to take on a different shape is possible by varying the number of off-circle roots. However, this will increase the ripple of the pattern (up to several decibels).

The array excitations $I_{n}$ are found by expanding the factors of (12) to (2). If desired, $S_{f}$ can be obtained by real valued excitations [21]. For asymmetric shapes, $I_{n}$ will always be complex.

4.4. Spreading the Collapsed Distributions. The reverse of collapsing a planar array is known as spreading the collapsed distribution. The strategy for spreading collapsed distributions depends on the structure of the planar array. For grid array structures, a system ofequations needs to be solved. Let the array factor of each distribution, collapsed on $\phi_{c}$, therefore be represented by

$$
F_{\phi_{c}}(\theta)=\sum_{l=1}^{L} I_{l} e^{j k d_{x^{\prime}} l \sin (\theta)},
$$

with $L$ the number of elements, the usual substitution $k=$ $2 \pi / \lambda$, and an interelement spacing $d_{x^{\prime}}$ according to Table 1 . When the $m n$th element of the planar array holds that

$$
n d_{x} \cos \left(\phi_{c}\right)-m d_{y} \sin \left(\phi_{c}\right)=d_{x^{\prime}} l
$$

this element contributes to the $l$ th element of the equivalent linear array. That is, (14) entails that the value $I_{l}$ is a summation of all the excitations $I_{m n}$ from the planar array of which their position $\left(x_{m n}, y_{m n}\right)$ collapses onto element $l$ of the linear array, as illustrated in Figure 8. One can thus write the equation

$$
\sum_{m=1}^{M} \sum_{n=1}^{N} \epsilon_{m n} I_{m n}=I_{l}
$$

with

$$
\epsilon_{m n}= \begin{cases}1, & \text { if } \frac{n d_{x} \cos \left(\phi_{c}\right)-m d_{y} \sin \left(\phi_{c}\right)}{d_{x^{\prime}}}=l \\ 0, & \text { otherwise. }\end{cases}
$$

By applying (15) to every element in the collapsed distribution, a system of equations is obtained. The complete system of equations is formed by applying (15) to the excitations $I_{l \phi_{c}}$ from every collapsed distribution

$$
\sum_{m=1}^{M} \sum_{n=1}^{N} \epsilon_{m n} I_{m n}=I_{l \phi_{c}}
$$

Denoting this as

$$
\mathbf{A x}=\mathbf{b},
$$

the coefficients of $\mathbf{A}$ represent $\epsilon_{m n}$, $\mathbf{b}$ the excitations $I_{l \phi_{c}}$ found for the collapsed distributions, and $\mathbf{x}$ the unknown excitations $I_{m n}$ of the planar array.

If $\mathbf{A}$ is square, the unknown $\mathbf{x}$ values can simply be found by matrix inversion. For underdetermined systems (or when the result deviates too much from the desired response), additional collapsed distributions should be added if possible. However, overdetermination is most likely to occur. Solving overdetermined systems is accomplished in a least squares sense, minimizing the norm $\|\mathbf{A x}-\mathbf{b}\|^{2}$. Hence the collapsed distribution principle will yield an approximate pattern shape in most cases.

Based on a system of equations containing the collapsed distributions from Table 1, shaping the SHA-169's pattern to $S_{f}$ yields the pattern depicted in Figure 9. This pattern clearly displays a very smooth shaped region again, albeit with hexagonal boundaries. Within a $\Delta \theta=30^{\circ}$ span, the ripple does not exceed $0.58 \mathrm{~dB}$, which is deemed well within the tolerance for shaped-pattern tracking based on simulations as described in [5].

\section{Asymmetric Collapsed Distributions}

Because the assumption of quadrant symmetry (as used in $[12,13])$ cannot be satisfied for $S_{r x}$ and $S_{r y}$, synthesizing 


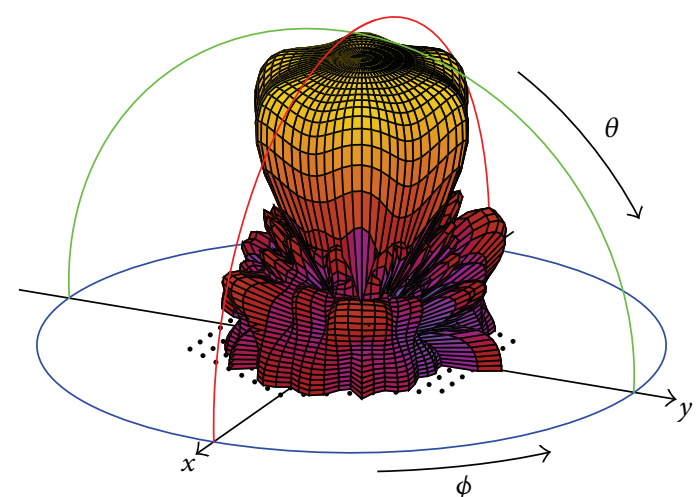

FIgURE 9: SHA-169 pattern shaped to $S_{f}$ using the collapsed distribution principle.

these shapes is not possible with just the steps described in Section 4 . The adjustment needed for asymmetric patterns involves specific placement (i.e., balancing) of the roots in (12) and applying a common normalization to the power pattern of the individual collapsed distribution.

5.1. Common Excitation Normalization. The collapsed distribution responses of a quadrant symmetric $[13,22]$ beam pattern such as $S_{f}$ are implicitly normalized, as they are all (practically) the same. Without such symmetry, a common normalization point needs to be agreed upon. Because every collapsed distribution response is guaranteed to intersect at the zenith, this is the most ideal place for normalization. Let the excitations $\stackrel{\circ}{I}_{l}$ from (13) therefore be adjusted by

$$
I_{l}=\frac{\left|\check{I}_{l}\right|}{\left|F_{\phi_{c}}\left(0^{\circ}\right)\right|} e^{j \arg \left(I_{l}\right)},
$$

such that $20 \log _{10}\left|F_{\phi_{c}}\left(0^{\circ}\right)\right|=0 \mathrm{~dB}$, as seen in Figure 10. This is the reason why $S_{r x}$ and $S_{r y}$ were defined with the $0 \mathrm{~dB}$ point at $\theta=0^{\circ}$ (Section 4.3). In many cases, including the ramp-shaped patterns, this power normalization will be sufficient. However, true alignment of the collapsed distributions requires slightly more elaborate measures. These are detailed in Appendix B.

5.2. Root Balancing. The meaning of root balancing is explained in the following. To this end, the roots of (12) must be written as

$$
w_{n}=e^{a_{n}+j b_{n}} .
$$

In case $a_{n} \neq 0$, the root lies off the unit circle [19]. One of the advantages of using (12) is that, for the power pattern, it does not matter whether $w_{n}$ lies outside $\left(+a_{n}\right)$ or inside $\left(-a_{n}\right)$ the unit circle. A pattern that has $N_{1}$ roots, where $a_{n} \neq 0$, gives rise to $2^{N_{1}}$ combinations of roots inside and outside the unit circle. In (12), expanding these different root combinations leads to different sets of excitations [19]. Often this feature is used to minimize the dynamic range between the excitations

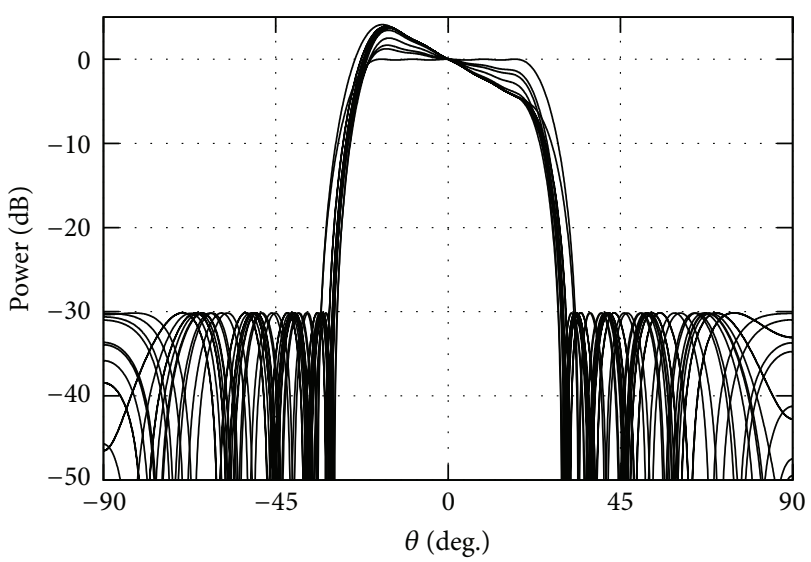

FIGURE 10: Collapsed $S_{r x}$ responses with their center normalized to $0 \mathrm{~dB}$.

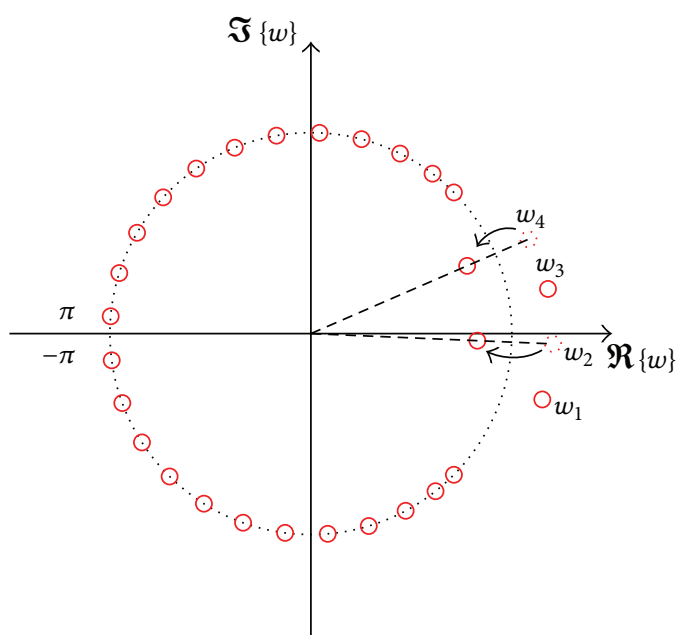

FIGURE 11: Balancing the root positions for $S_{r x}\left(0^{\circ}, \theta\right)$.

(i.e., $\max \left\{\left|I_{n}\right|\right\} / \min \left\{\left|I_{n}\right|\right\}$ ), to alleviate problems with mutual coupling [23].

By default, Orchard-Elliott synthesis will give the designer $N_{1}$ roots outside the circle (Figure 11). If the corresponding excitations are used in the collapsed distribution principle, the result will be a pattern with deep nulls inside the shaped region. Figure 12 shows this for several $\phi$-cuts of the $S_{r x}$ shape, which is clearly unacceptable for tracking purposes.

Expansion of (20) and solving of (17) are fast. When different root combinations are tried exhaustively, it is very likely that a much better result will be found using a different set of roots, for example, the set presented in Figure 11. By moving two out of four roots outside the circle to their opposing angular reciprocal position, the response improves considerably. This strategy has been applied to the collapsed distributions of various different pattern shapes, and it is observed that a balanced set of roots always produces the best result in terms of ripple. Such a balanced set has $\left\lfloor N_{1} / 2\right\rfloor$ roots outside the circle and $\left\lceil N_{1} / 2\right\rceil$ roots inside the circle, or vice versa. In this particular example, one could also have chosen 


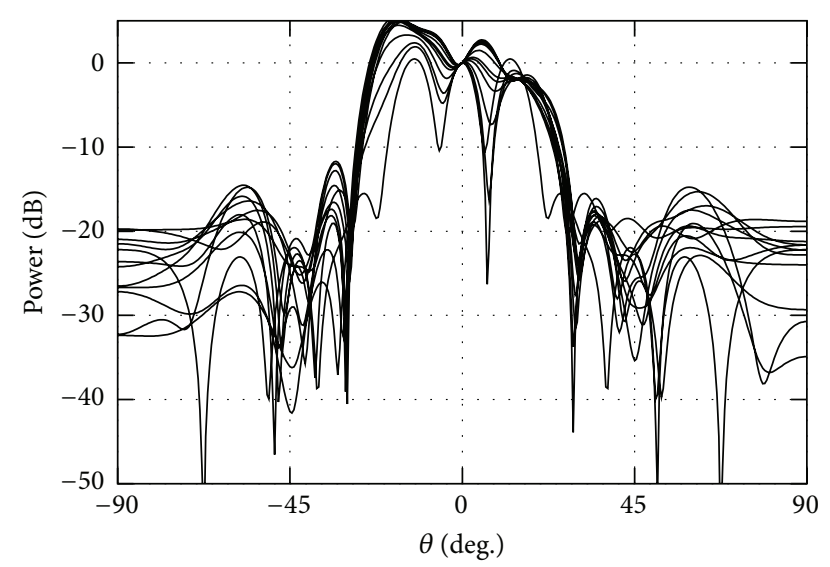

FIGURE 12: Response after spreading collapsed distributions, without balancing the polynomial roots $\left(\forall n \in 1, \ldots, 4 \mid a_{n}>0\right)$.

to move $w_{1}$ and $w_{2}$ instead of $w_{2}$ and $w_{4}$ (Figure 11). The resulting pattern will then exhibit a slightly higher ripple, but the dynamic range of the excitations is lower. A compromise needs to be made.

It must be noted that the above is based purely on the evidence obtained by synthesizing different pattern shapes. No proof is given that the balanced roots condition is sufficient for every possible pattern.

5.3. Expanding Large Collapsed Distributions. Another problem that may be encountered, which is unrelated to symmetry but not addressed elsewhere, is the size of collapsed distributions. Collapsed distributions tend to become large in terms of Orchard-Elliott synthesis, which may lead to numerical errors. Appendix C covers this subject in detail.

5.4. Results. Having taken the necessary precautions, spreading the collapsed distributions of $S_{r x}$ and $S_{r y}$ gives patterns as shown in Figures 13 and 14, respectively. The ripple in $S_{r x}$ does not exceed $0.68 \mathrm{~dB}$ and the ripple of $S_{r y}$ is at most $0.57 \mathrm{~dB}$. Similar to $S_{f}$, both were found to be sufficiently small for tracking purposes.

\section{Steering and Shape Preservation}

Having established the three "basic" beam patterns, the questions that remain are how they will behave under different steering angles and whether they are indeed suitable for tracking purposes. It will first be demonstrated that, because of its higher degree of rotational symmetry, the hexagonal array is much more capable of retaining the shape of the patterns when steered.

6.1. Steering Hexagonal Beam Patterns. Recall that the (hexagonally) shaped beam's orientation can be changed "lossless" to any of the pseudo axes, by a different mapping of the excitations to the element positions. Such a mapping is easily realized when the excitations are organized in a matrix $\mathbf{W}$, with indexing that matches Figure 6 . Let every $m$ th row

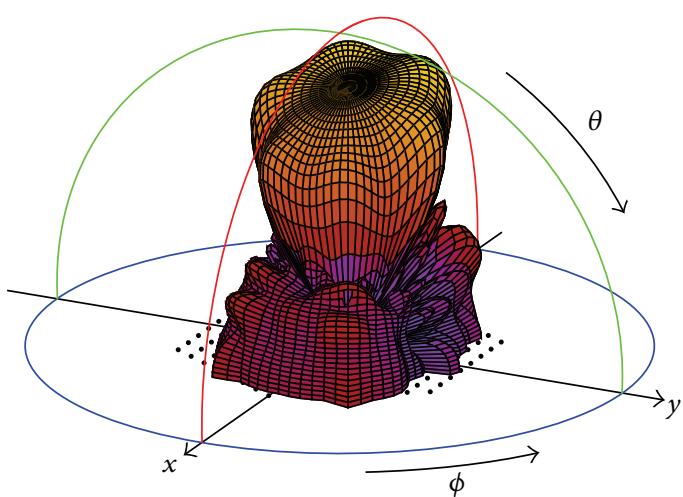

FIGURE 13: SHA-169 pattern shaped to $S_{r x}$.

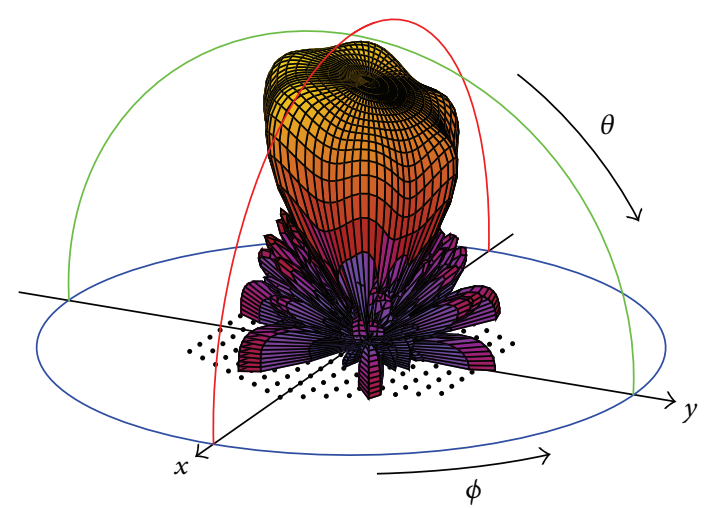

FIGURE 14: SHA-169 pattern shaped to $S_{r y}$.

where $m<\lceil M / 2\rceil$ be padded with leading zeros and every $m$ th row where $m>\lceil M / 2\rceil$ with trailing zeros (i.e., dummy values). The transpose $\mathbf{W}^{T}$ will rotate the patterns' shape by $300^{\circ}$ (and flip it over the $x$-axis), as illustrated by the following example:
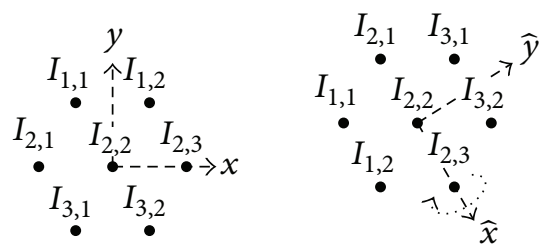

$$
\left[\begin{array}{ccc}
\mathbf{0} & I_{1,1} & I_{1,2} \\
I_{2,1} & I_{2,2} & I_{2,3} \\
I_{3,1} & I_{3,2} & \mathbf{0}
\end{array}\right]^{T}
$$$$
\left[\begin{array}{ccc}
\mathbf{0} & I_{2,1} & I_{3,1} \\
I_{1,1} & I_{2,2} & I_{3,2} \\
I_{1,2} & I_{2,3} & \mathbf{0}
\end{array}\right]
$$

Rotation to any of the other pseudo axes is simply derived by mirror operations. To change the elevation angle, or steer to a $\phi$ direction off the principal axes, every $m n$th excitation still has to be weighed according to expression (6). However, the mapping greatly reduces the need for $\phi$ steering outside the pattern's axes of reference, thus keeping the angular coverage more consistent. Given their coverage (i.e., beamwidth), the patterns shown here will not need to be steered more than $10^{\circ}$ away from the pseudo axes. This makes $\phi=50^{\circ}$ (i.e., $60^{\circ}-10^{\circ}$ ) 


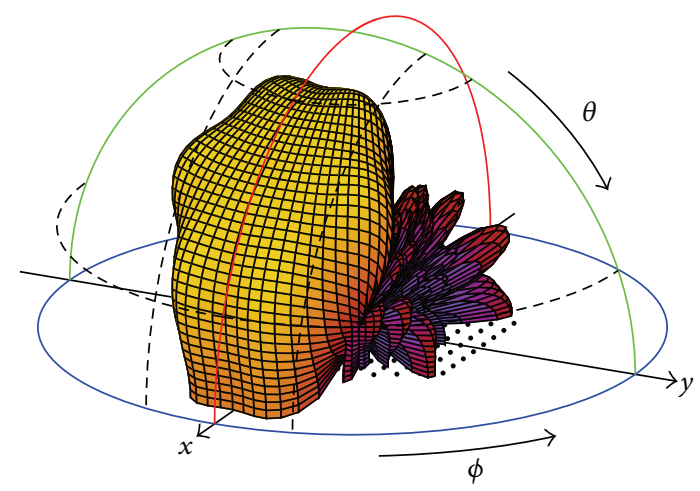

Figure 15: Coverage after steering the SHA-169 $S_{f}$ pattern to $\left(0^{\circ}, 35^{\circ}\right)$.

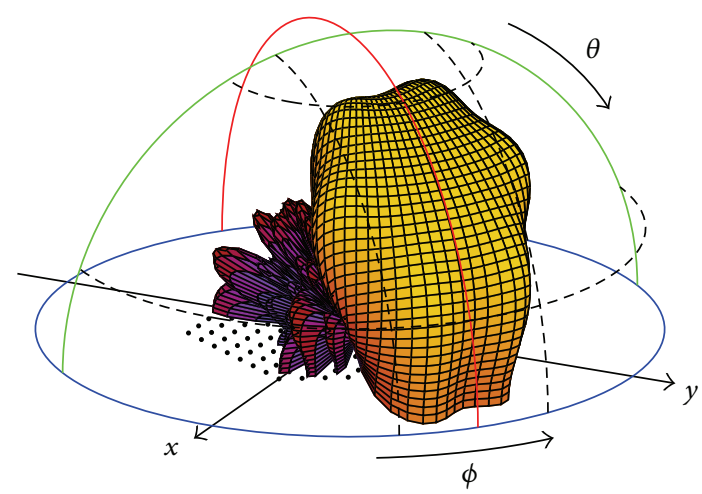

Figure 16: Coverage after steering the SHA-169 $S_{f}$ pattern to $\left(50^{\circ}, 35^{\circ}\right)$.

one of the larger steering angles that will be required. Figures 15 and 16 show that the shape of the pattern is very similar when subject to $\phi=0^{\circ}$ or $\phi=50^{\circ}$, as intended.

6.2. Tracking Considerations. To assess whether the patterns remain sufficiently consistent for tracking whilst steered, the combinations $S_{f}$ with $S_{r x}$ and $S_{f}$ with $S_{r y}$ are steered to $50^{\circ}$ azimuth and $45^{\circ}$ elevation. In terms of shape retention, this steering angle is representative for the worst case needed in covering the entire upper $(\phi, \theta)$ hemisphere with respect to the array center. From the azimuth and elevation planes, respectively, crosscuts are provided in Figures 17 and 18. Figure 17 depicts $S_{r x}$ as being flat in the plane parallel to the page and ramp-shaped perpendicular to the page, while Figure 18 shows $S_{r y}$ in the same manner. Herein it can be seen that the power differences between the flat and ramp-shaped patterns are quite consistent within a $20^{\circ}$ by $30^{\circ}$ coverage area, both in the $\phi$ and $\theta$ direction. Based on this observation, the ideas from Section 2 can directly be carried over to the $2 \mathrm{D}$ domain. The steering angle can simply be updated based on the power levels associated with the boundaries of the $20^{\circ}$ by $30^{\circ}$ coverage area.

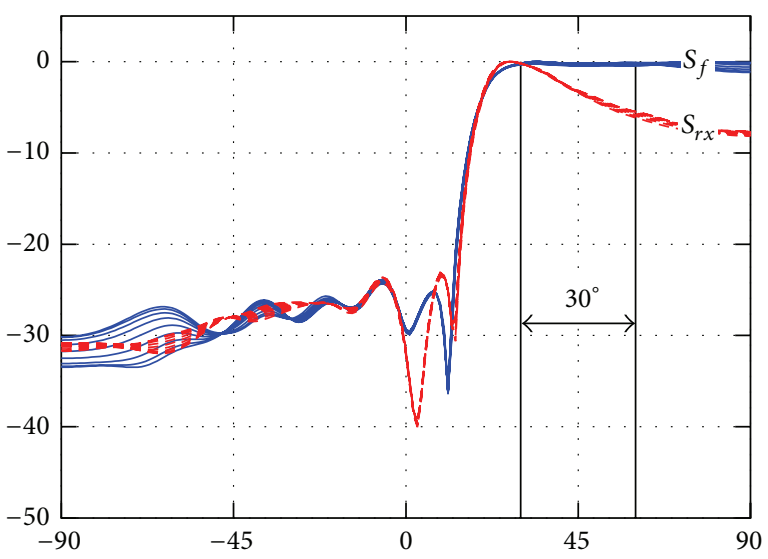

Figure 17: Azimuth crosscuts of the $S_{f}$ and $S_{r x}$ patterns steered to $\left(50^{\circ}, 45^{\circ}\right)$, covering the range $40^{\circ} \leq \phi \leq 60^{\circ}$ in the azimuth plane $\left(20^{\circ}\right.$ in Figure 18).

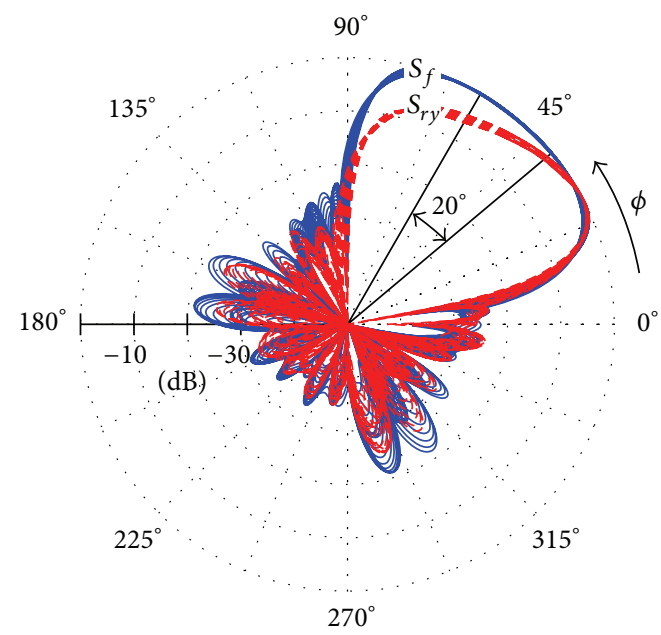

FIGURE 18: Elevation crosscuts of the $S_{f}$ and $S_{r y}$ patterns, steered to $\left(50^{\circ}, 45^{\circ}\right)$ and covering the elevation range $30^{\circ} \leq \theta \leq 60^{\circ}\left(30^{\circ}\right.$ in Figure 17).

\section{Future Work}

If the synthesis procedure is extended further to take the EM properties of nonideal antennas into account, a closer correspondence between the synthesized patterns and their physical implementation can be achieved. The two most influential factors are the directivity of the antenna elements (i.e., element factor [6]) and mutual coupling. For linear synthesis, compensation of the element factor has been addressed in [17]. It is expected that this technique can be carried over to the collapsed distribution principle directly by employing it during linear synthesis for the collapsed distributions. It is not known whether the mutual coupling effect can be compensated in the same way. At least for the linear techniques reported in $[24,25]$ there is not a straightforward extension for collapsed distributions.

In terms of tracking, a detailed plan needs to be developed. This encompasses determining precisely what steering 
angles (patterns) are needed for efficient $(\phi, \theta)$ coverage and defining how the steering angles should be updated. A simulation similar to the one carried out in [5], but with more accurate channel characteristics and for 2D position tracking, is planned. Furthermore, although the examples encountered so far suggest that the balanced roots condition works for asymmetric patterns, this still needs to be proven. It would be beneficial for the method if actual proof is found. Finally it may also be interesting to investigate how far the array size can be scaled down. Based on the findings for linear arrays [5], it is expected that a 61-element SHA might work well.

\section{Conclusion}

A planar array synthesis method for asymmetrically shaped beam patterns with low ripple has been established. It has been shown that when using a hexagonal array geometry, these patterns can be steered without significantly changing their shape. This is particularly important for shapedpattern tracking. The synthesis procedure is based on the quasi-analytical method of collapsed distributions, which was previously limited to rotational or quadrant symmetric beam patterns. In order to synthesize asymmetric shapes, the roots found with Orchard-Elliott synthesis need to be balanced and all the individual collapsed distributions need to be normalized explicitly to a common point. The resulting patterns, on average, feature a ripple of $0.6 \mathrm{~dB}$. This is well within the bounds for shaped-pattern tracking.

\section{Appendices}

\section{A. Simulated Annealing and Genetic Algorithms}

Some preliminary testing was conducted on the use of Simulated Annealing (SA) and the Genetic Algorithm (GA) for synthesis of low ripple 2D beam patterns. In both techniques, the cost function (or fitness function) that is minimized by the algorithm is the most important parameter for successful optimization. Most popular is the root-mean-square of the relative error

$$
E_{\mathrm{Q}}=\left(\frac{1}{Q} \sum_{q=1}^{\mathrm{Q}} h_{q}\left|e_{q}\right|^{2}\right)^{1 / 2},
$$

computed at $Q$ points in the pattern, where $e_{q}$ is the difference between the actual pattern and the desired one. A weighting function $h_{q}$ can optionally be employed to emphasize various aspects (or areas) of the pattern. In the linear array case, such a function typically results in a pattern as depicted in Figure 19.

This result was obtained with the MATLAB Global Optimization Toolbox, using GA optimization. A population size of $6 \mathrm{~N}$ was chosen with six elites per generation and a crossover fraction of 0.8 . Termination was forced after the 10000th generation.

The relatively high sidelobe region is the result of how $e_{q}$ is determined. As shown in Figure 19, this was done based

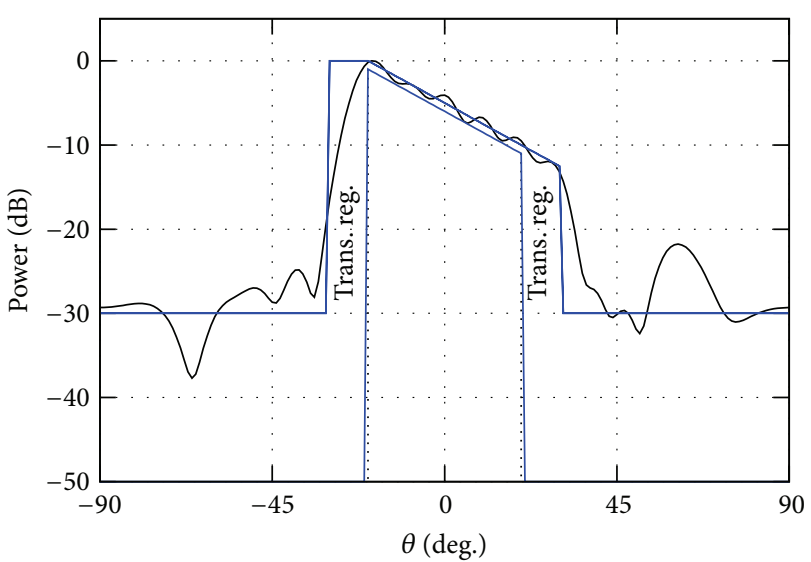

FIGURE 19: Beam pattern of a $N=15$ element linear array, shaped to $S_{r x}\left(0^{\circ}, \theta\right)$ using the real encoded GA of MATLAB.

on a mask with upper and lower bounds [9] rather than actual shaping function $S$. One advantage of this is that zero costs can be associated with areas of the pattern that produce large errors but are not immediately interesting for a low ripple. Examples of this are the transient regions and the nulls between sidelobes.

Another common cost function is

$$
E_{\mathrm{Q}}=\sum_{q=1}^{\mathrm{Q}} \log \left|\frac{S_{q}}{A F_{q}}\right|
$$

with $S_{q}$ the desired power at $q$ and $A F_{q}$ the actual power. This function is mainly effective for creating deep nulls at specified locations. A hybrid of (A.1) and (A.2) can also be useful [7]. All the above cost functions (based on RMS errors, absolute relative errors, and square errors) have been evaluated in order to improve the ripple from Figure 19 without merit. A ripple below $0.5 \mathrm{~dB}$ could not be obtained by directly manipulating the array excitations. (Better results can be found in literature, but these are based on the Schelkunoff representation and are thus unsuitable for planar arrays.)

For planar arrays, the problem complexity increases drastically because of the many degrees of freedom involved. As a result, the ripple of the obtained patterns is even worse, with nulls frequently dipping below $-20 \mathrm{~dB}$ in the shaped region. Besides different fitness functions, attempts to improve the ripple include increasing and decreasing the population size; performing several short runs instead of a longer one; and more dense sampling $(q)$ in the shaped region. However, even with a pool of six threads working on the problem in parallel, 72 hours of optimization did not lead to a desirable pattern. This indicates that it will be extremely hard to find low ripple shaped patterns using GAs.

Simulated Annealing performs similarly when used as an independent technique. However, [22] reports that SA can also be used as an additional optimization step after, for example, collapsed distributions. By seeding it with a known good set of excitations, SA can control the ripple minima and maxima while lowering the dynamic range of the excitations. 
This has not yet been tried for the patterns presented in this paper.

\section{B. Collapsed Distributions Normalization}

It is assumed that the reader is familiar with the basic principles of Orchard-Elliott synthesis. There is a shaping function $S$ and the power pattern $G(\psi)$ given as

$$
\begin{aligned}
G(\psi)= & \sum_{n=1}^{N-2} 10 \log _{10}\left[1-2 e^{a_{n}} \cos \left(\psi-b_{n}\right)+e^{2 a_{n}}\right] \\
& +10 \log _{10}[2(1+\cos (\psi))]+C_{1},
\end{aligned}
$$

wherein $\psi=\left(2 \pi d_{x^{\prime}} / \lambda\right) \sin (\theta)$ and the roots of (12) are decomposed into $w_{n}=e^{a_{n}+j b_{n}}$. The objective is to minimize $G-S$, which is done by perturbing $a_{n}$ and $b_{n}$ and evaluating $\partial G(\psi) / \partial a_{n}$ or $\partial G(\psi) / \partial b_{n}$ at key points (i.e., the extrema) in the pattern. In [19], $S$ is defined relative to $\psi_{0}$ so that $G\left(\psi_{0}\right)=$ $\max \{G(\psi)\}$ (i.e., the peak of the main beam). The constant $C_{1}$ in (B.1) normalizes $G\left(\psi_{0}\right)$ to $0 \mathrm{~dB}$. Defining $S$ in such a way becomes problematic when $G(\psi)$ is not symmetric, because in that case $G\left(\psi_{0}\right)$ is not necessarily $0 \mathrm{~dB}$ after being normalized by (19). The most notable consequence is that the sidelobes will not arrive at the specified power level. However, the shaped region is also affected to some extent. Without symmetry, the correct way of specifying $S$ is relative to the MRA, say $\psi_{m}$. In the interest of obtaining the smallest possible ripple, this was also done for $S_{r x}$ and $S_{r y}$.

To specify $S$ relative to $\psi_{m}, \psi_{m}$ needs to be known. Unfortunately it is not possible to predict exactly where $\psi_{m}$ will be, because the shape of $G$ changes as it converges on $S$. A good estimate can be found by making use of the fact that the shaped region stays confined between two particular roots. Let the pattern be organized as in [19]. The first $N_{1}$ roots of $G$ are then positioned in the shaped region, confining the latter between $-\pi$ and $b_{N_{1}+1}$. Consequently $\psi_{m}=\left(b_{N_{1}+1}-\pi\right) / 2$, as identified in Figure 20. The balance between the left and right transient regions (see Figure 19) [9] determines how accurate the estimate will be. For many synthesizable shapes, these transient regions are comparable in size.

In [19] a constant $C_{2}$ is associated with $S$ to compensate for small errors made in normalizing $G\left(\psi_{0}\right)$ to $0 \mathrm{~dB}$. If $G\left(\psi_{0}\right)$ were to end up at, for example, $0.5 \mathrm{~dB}$, then $G$ should be shaped to $S-0.5$ in the next iteration. Since $S$ is now defined relative to $\psi_{m}$, this $C_{2}$ constant should of course also be computed such that $G\left(\psi_{m}\right)$ goes to $0 \mathrm{~dB}$. In Figure 20 this has been illustrated as $S=S_{0}-C_{2}$. The $C_{2}$ normalization should not be confused with (19), which is still necessary prior to solving the system of equations.

\section{Large Collapsed Distributions}

For many of the entries in Table 1, straightforward multiplying out the right hand side of (12) can no longer be done in reasonable time. Let the roots of (12) be collected in a vector $\mathbf{w}$. The coefficients $\mathbf{c}$ of the polynomial (i.e., excitations) can then be found much faster with the "Summation Algorithm" $(\operatorname{poly}(\mathbf{w})$ in MATLAB).

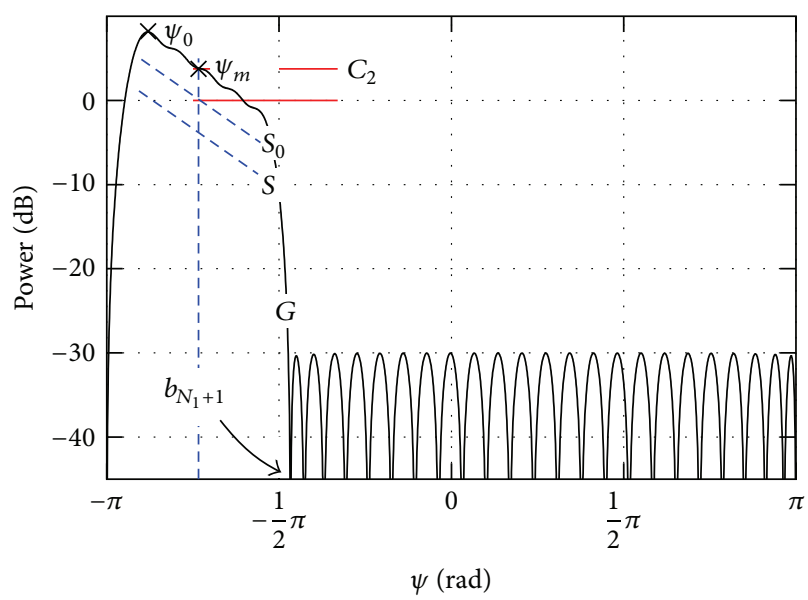

FIGURE 20: Shaping $G$ (4th iteration) to $S_{r x}\left(0^{\circ}, \theta\right)$ relative to the MRA instead of $\psi_{0}$.

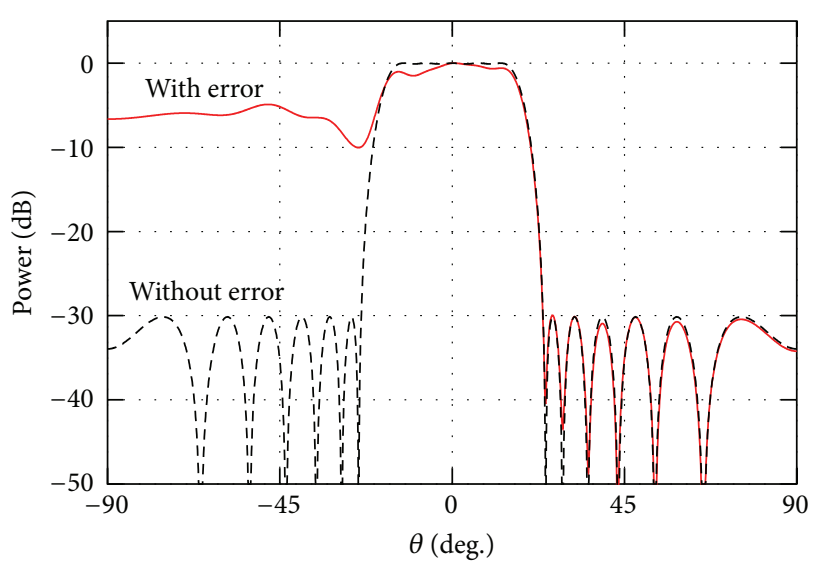

FIGURE 21: Rounding errors made during root to excitation conversion may lead to an unusable pattern shape.

However, in spite of the relatively good numerical stability, this algorithm does suffer from rounding errors [26]. Results may vary depending on the set of roots, but, on several occasions where $N \gtrsim 57$, standard IEEE-754 double precision floating-point arithmetic was found to be inadequate. Figure 21 shows the conversion of a flat-top pattern for $N=$ 71 (i.e., $\phi_{c}=16.01^{\circ}$ ), to illustrate the effect these errors can lead to. Clearly this response is not suitable for further use.

An obvious solution to this problem is to use higher precision arithmetic. All the collapsed distributions discussed in this work were indeed expanded using quad precision arithmetic, available in the Advanpix arbitrary precision toolbox for MATLAB [27]. However, there exists a solution that does not require special tools. Wilkinson's polynomial showed that in particular the coefficients in the center of the polynomial are affected by rounding errors [28]. Furthermore, the order in which the roots in $\mathbf{w}$ occur does not affect the resulting coefficients $\mathbf{c}$. It does determine which coefficients are going to be affected most by the rounding errors. Since large collapsed distributions often produce a spacing $d_{x^{\prime}}<0.5 \lambda$, their visible region is smaller than 


$$
\begin{aligned}
& \mathbf{c}[1] \leftarrow 1 \\
& \mathbf{c}[2: n] \leftarrow 0 ; \\
& \text { for } i \leftarrow 1 \text { to } N \text { do } \\
& \quad \mathbf{c}[2: i+1]=\mathbf{c}[2: i+1]-\mathbf{w}[i] * \mathbf{c}[1: i] \\
& \text { end }
\end{aligned}
$$

Algorithm 1: Summation Algorithm [26].

the physical visible region (i.e., $-90^{\circ}, \ldots, 90^{\circ}$ ). There is thus a good probability that one can find $\iota$ so that the differently ordered roots

$$
\mathbf{w}(i)=\stackrel{\circ}{\mathbf{w}}((i+\iota) \bmod (N-1))
$$

converted by Algorithm 1 yield excitations for which only the parts of the pattern outside the visible region are affected by errors. These excitations can be used in the system of equations. Should the visible region not be smaller than $180^{\circ}$, then it is still possible to exploit the fact that the excitation furthest away from the center affects the pattern's shape the least [29].

\section{Conflict of Interests}

The authors declare that there is no conflict of interests regarding the publication of this paper.

\section{Acknowledgments}

The authors would like to thank Julio Illade-Quinteiro and Fransisco Ares-Pena from the University of Santiago de Compostela for their assistance in understanding the principle of collapsed distributions. This work is part of the SOWICI research project (647.000.005), which is financed by the Netherlands Organization for Scientific Research.

\section{References}

[1] K. Chandra, Z. Cao, T. Bruintjes et al., "mCRAN: a radio access network architecture for $5 \mathrm{G}$ indoor communications," in Proceedings of the IEEE International Conference on Communication Workshops (ICC '15), pp. 300-305, IEEE, London, UK, June 2015.

[2] J. G. Andrews, S. Buzzi, W. Choi et al., "What will 5G be?" IEEE Journal on Selected Areas in Communications, vol. 32, no. 6, pp. 1065-1082, 2014.

[3] B. Allen and M. Ghavami, Adaptive Array Systems: Fundamentals and Applications, Wiley-Blackwell, 1st edition, 2005.

[4] S. Chandran, Adaptive Antenna Arrays: Trends and Applications, chapter 3, Springer, 1st edition, 2004.

[5] T. M. Bruintjes, A. B. Kokkeler, G. Karagiannis, and G. J. Smit, "Using shaped beam patterns for tracking," IEEE Transactions on Antennas and Propagation, vol. 62, no. 12, pp. 6496-6501, 2014.

[6] H. L. van Trees, Optimum Array Processing (Detection, Estimation, and Modulation Theory, Part IV), Wiley-Interscience, 1st edition, 2002.
[7] F. J. Villegas, "Parallel genetic-algorithm optimization of shaped beam coverage areas using planar $2-D$ phased arrays," IEEE Transactions on Antennas and Propagation, vol. 55, no. 6, pp. 1745-1753, 2007.

[8] D. Gies and Y. Rahmat-Samii, "Particle swarm optimization for reconfigurable phase-differentiated array design," Microwave and Optical Technology Letters, vol. 38, no. 3, pp. 168-175, 2003.

[9] O. M. Bucci, G. D’Elia, G. Mazzarella, and G. Panariello, "Antenna pattern synthesis: a new general approach," Proceedings of the IEEE, vol. 82, no. 3, pp. 358-371, 1994.

[10] L. I. Vaskelainen, "Constrained least-squares optimization in conformal array antenna synthesis," IEEE Transactions on Antennas and Propagation, vol. 55, no. 3, pp. 859-867, 2007.

[11] H.-T. Chou, N.-N. Wang, H.-H. Chou, and J.-H. Qiu, "An effective synthesis of planar array antennas for producing nearfield contoured patterns," IEEE Transactions on Antennas and Propagation, vol. 59, no. 9, pp. 3224-3233, 2011.

[12] F. Ares-Pena, J. A. Rodriguez-Gonzalez, and A. Vieiro, "Efficient footprint patterns obtained by spreading out collapsed distributions," in Proceedings of the IEEE Antennas and Propagation Society International Symposium, vol. 1, pp. 498-501, IEEE, Montreal, Canada, July 1997.

[13] J. Illade-Quinteiro, J. A. Rodriguez-González, and F. AresPena, "Shaped-pattern synthesis by spreading out collapsed distributions," IEEE Antennas and Propagation Magazine, vol. 52, no. 6, pp. 110-114, 2010.

[14] J. Capon, "High-resolution frequency-wavenumber spectrum analysis," Proceedings of the IEEE, vol. 57, no. 8, pp. 1408-1418, 1969.

[15] A. Paulraj, R. Roy, and T. Kailath, "Estimation of signal parameters via rotational invariance techniques- esprit," in Proceedings of the 19th Asilomar Conference on Circuits, Systems and Computers, pp. 83-89, Pacific Grove, Calif, USA, November 1985.

[16] R. O. Schmidt, "Multiple emitter location and signal parameter estimation," IEEE Transactions on Antennas and Propagation, vol. 34, no. 3, pp. 276-280, 1986.

[17] T. M. Bruintjes, A. B. J. Kokkeler, G. Karagiannis, and G. J. M. Smit, "Shaped pattern synthesis for equispaced linear arrays with non-isotropic antennas," in Proceedings of the European Conference on Antennas and Propagation (EuCAP '15), pp. 1-5, Lisbon, Portugal, April 2015.

[18] R. S. Elliott, "Array pattern synthesis part II: planar arrays," IEEE Antennas and Propagation Society Newsletter, vol. 28, no. 2, pp. 4-10, 1986.

[19] H. J. Orchard, R. S. Elliott, and G. J. Stern, "Optimising the synthesis of shaped beam antenna patterns," IEEE Proceedings of Microwaves, Antennas and Propagation, vol. 132, no. 1, pp. 6368, 1985.

[20] S. A. Schelkunoff, "A mathematical theory of linear arrays," The Bell System Technical Journal, vol. 22, no. 1, pp. 80-107, 1943.

[21] Y. U. Kim and R. S. Elliott, "Shaped-pattern synthesis using pure real distributions," IEEE Transactions on Antennas and Propagation, vol. 36, no. 11, pp. 1645-1649, 1988.

[22] F. Ares-Pena, R. S. Elliott, and E. Moreno, "Design of planar arrays to obtain efficient footprint patterns with an arbitrary footprint boundary," IEEE Transactions on Antennas and Propagation, vol. 42, no. 11, pp. 1509-1514, 1994.

[23] R. S. Elliott and G. J. Stern, "A new technique for shaped beam synthesis of equispaced arrays," IEEE Transactions on Antennas and Propagation, vol. 32, no. 10, pp. 1129-1132, 1984. 
[24] H. Oraizi and M. Fallahpour, "Array pattern synthesis with mutual coupling consideration," in Proceedings of the International Symposium on Telecommunications (IST '08), pp. 77-82, Tehran, Iran, August 2008.

[25] C. Reck, U. Berold, and L.-P. Schmidt, "Automated synthesis of shaped beam antenna patterns with implied cross coupling," in Proceedings of the 4th European Conference on Antennas and Propagation (EuCAP '10), pp. 1-4, IEEE, Barcelona, Spain, April 2010.

[26] R. Rehman and I. C. F. Ipsen, "Computing characteristic polynomials from eigenvalues," SIAM Journal on Matrix Analysis and Applications, vol. 32, no. 1, pp. 90-114, 2011.

[27] Advanpix, "Multiprecision computing toolbox for matlab," 2015, http://www.advanpix.com/.

[28] J. H. Wilkinson, Rounding Errors in Algebraic Processes, Prentice-Hall, Englewood Cliffs, NJ, USA, 1st edition, 1963.

[29] Y. U. Kim and J. D. Nespor, "Shaped beam synthesis and conditional thinning for planar phased array," in Proceedings of the Digest Antennas and Propagation Society International Symposium (AP-S '96), vol. 2, pp. 802-805, IEEE, Baltimore, Md, USA, July 1996. 


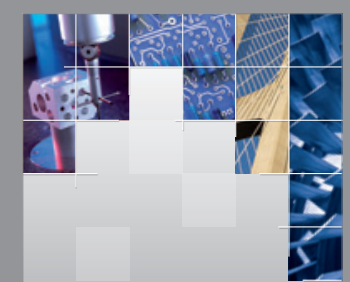

\section{Enfincering}
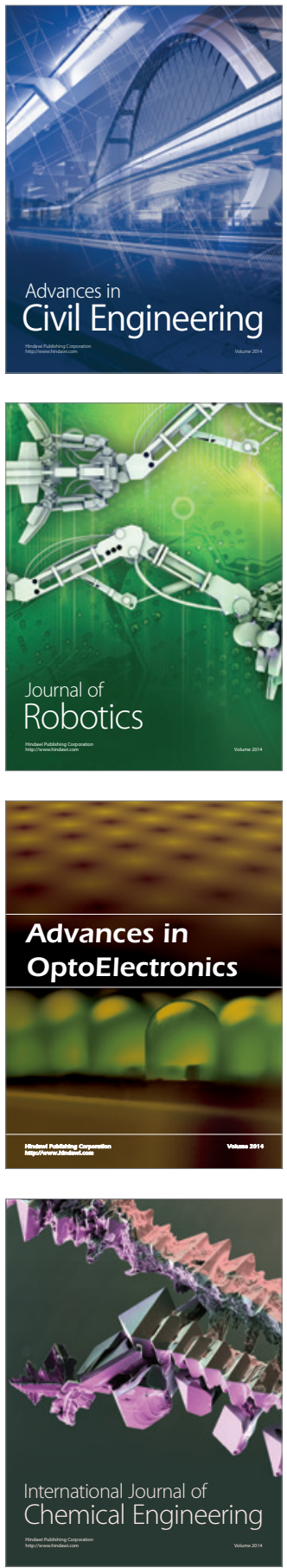

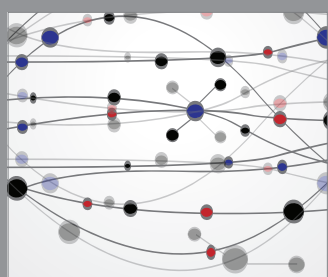

The Scientific World Journal

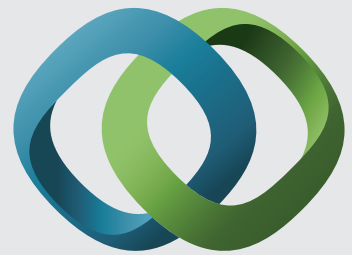

\section{Hindawi}

Submit your manuscripts at

http://www.hindawi.com
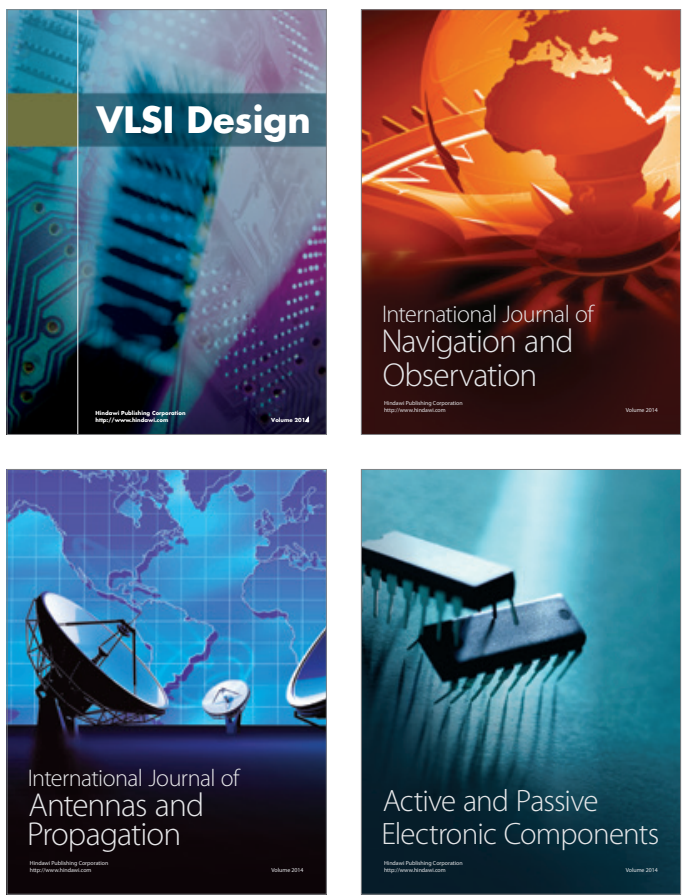
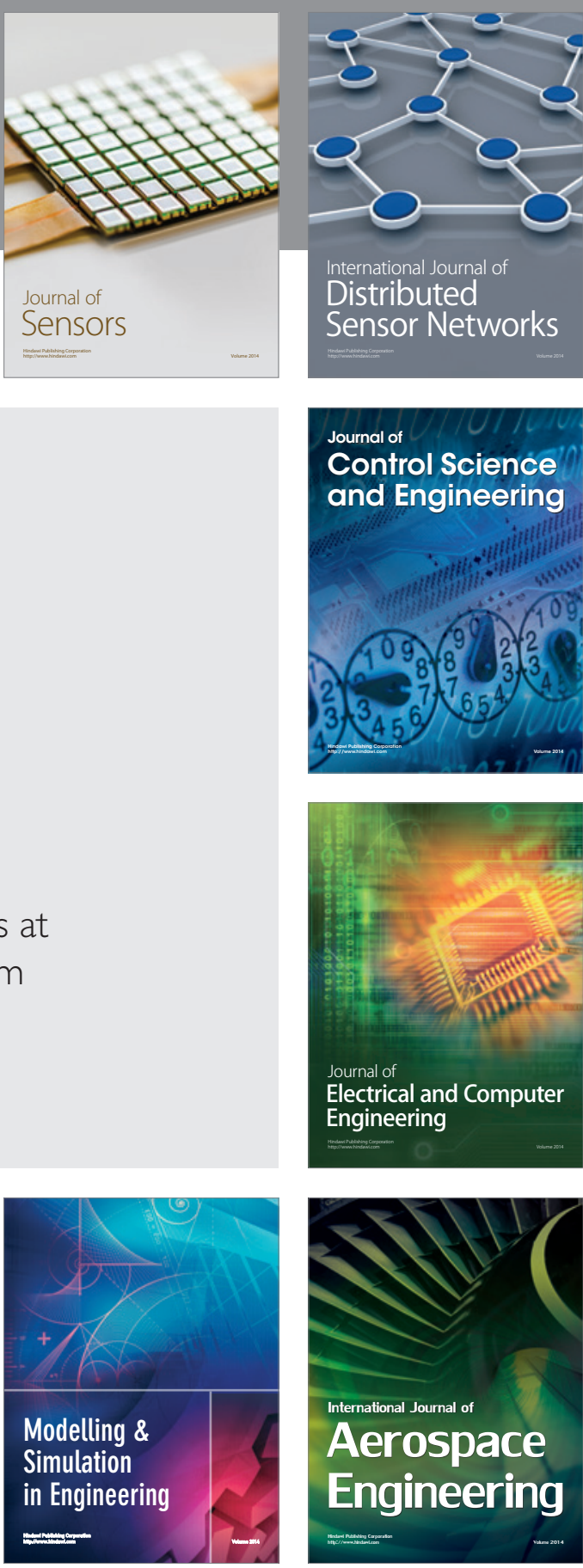

International Journal of

Distributed

Sensor Networks

Journal of

Control Science

and Engineering
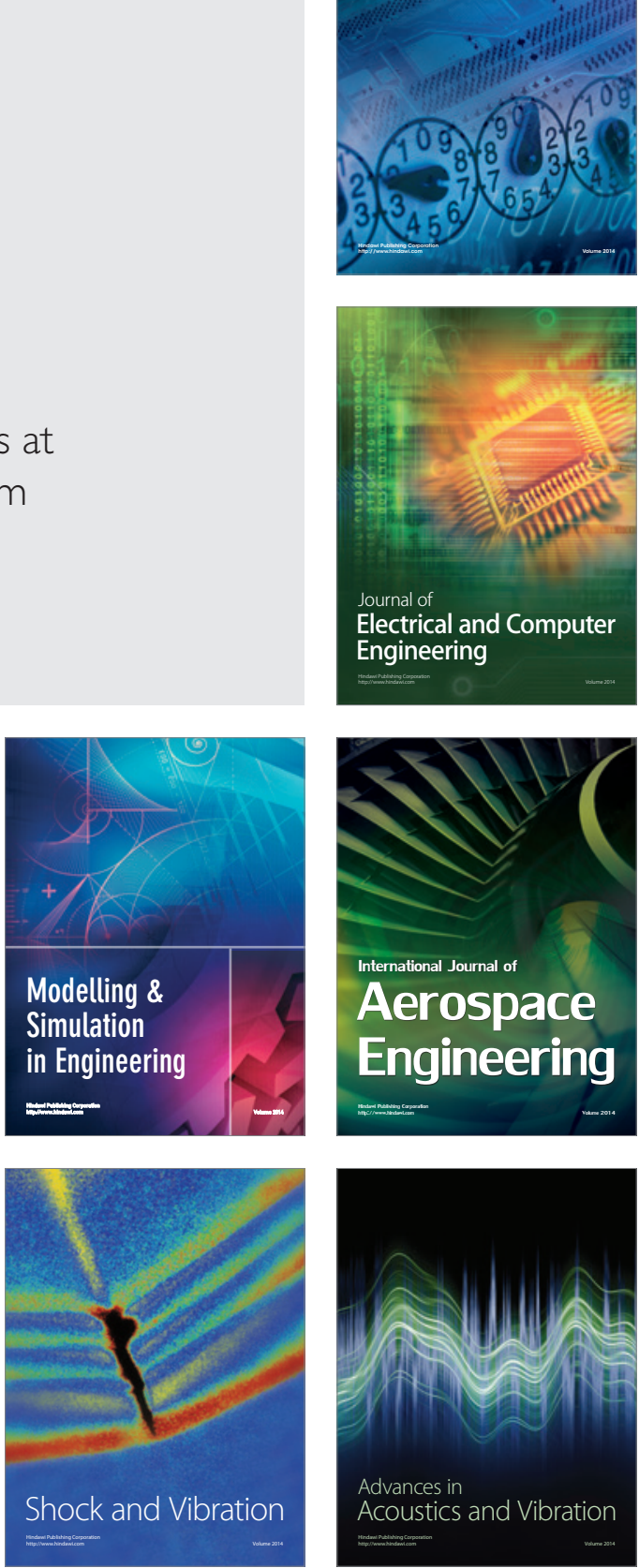